\title{
Use of BioSense for Rapid Assessment of the Safety of Medical Countermeasures
}

\author{
Ralph J. Coates* and Kathleen Gallagher \\ Division of Notifiable Diseases and Healthcare Information, Centers for Disease Control and Prevention, Atlanta, GA, USA
}

\section{Objective}

To conduct an initial examination of the potential use of BioSense data to monitor and rapidly assess the safety of medical countermeasures (MCM) used for prevention or treatment of adverse health effects of biological, chemical, and radiation exposures during a public health emergency.

\section{Introduction}

BioSense is a national human health surveillance system for disease detection, monitoring, and situation awareness through near realtime access to existing electronic healthcare encounter information, including information from hospital emergency departments (EDs). MCM include antibiotics, antivirals, antidotes, antitoxins, vaccinations, nuclide-binding agents, and other medications. Although some MCM have been extensively evaluated and have FDA approval, many do not (1). Current FDA and CDC systems that monitor drug and vaccine safety have limited ability to monitor MCM safety, and in particular to conduct rapid assessments during an emergency (1).

\section{Methods}

To provide a preliminary assessment of the use of BioSense for this purpose, we reviewed selected publications evaluating the use of electronic health records (EHRs) to monitor safety of drugs and vaccinations (medications), focusing particularly on systematic reviews, reviewed BioSense data elements, and consulted with a number of subject matter experts.

\section{Results}

More than 40 studies have examined use of EHR data to monitor adverse effects (AEs) of medications using administrative, laboratory, and pharmacy records from inpatient- and out-patient settings, including EDs (2-4). To identify AEs, investigators have used diagnostic codes; administration of antidotes, laboratory measures of drug levels and of biologic response, text searches of unstructured clinical notes, and combinations of those data elements. BioSense ED data include chief complaint text, triage notes, text diagnosis, as well as diagnostic and medical procedure codes.

Investigations used a variety of study designs in various populations and settings; examined a wide range of medications, vaccinations, and AEs; and developed a diverse set of analytic algorithms to search EHR data to detect and signal AEs (2-4). Most research has been done on FDA-approved medications. Most studies used EHR data to identify individuals using specific medications and then searched for potential AEs identified from previous research. None of the studies investigated use of EHR data to monitor safety when records of an individual's medication use could not be linked to that individual's records of AEs. BioSense data could be used for AE detection, but linking AEs to MCM use would require follow-back investigation. Since there is limited research on AEs of some MCM, there would be limited information to guide identification of potential AEs.

Performance characteristics of the AE monitoring systems have been mixed with reported sensitivities ranging from $40-90 \%$; specificities from $1 \%$ to $90 \%$, and positive predictive values from $<1 \%$ to $64 \%$, depending on the medication, AE and other characteristics of the study $(2,4)$. However, the small numbers of studies with common characteristics has limited the ability of reviewers to determine which types of systems have better performance for different medications and AEs.

Some experts suggest that data in BioSense, might contribute to safety surveillance of MCM. They also caution that poor predictive values and high rates of false positives reported in the literature raise concerns about burden to those conducting investigations in response to $\mathrm{AE}$ alerts, particularly in the context of a public health emergency.

\section{Conclusions}

These findings suggest that BioSense data could potentially contribute to rapid identification of safety issues for MCM and that some methods from published research could be applicable to the use of BioSense for this purpose. However, such use would require careful development and evaluation.

\section{Keywords}

BioSense; countermeasures; safety; monitoring; medical

\section{References}

1. National Biodefense Science Board. Where are the countermeasures? Protecting America's health from CBRN threats. U. S. Department of Health and Human Services, March, 2010.

2. Forster AJ, et al. A systematic review to evaluate the accuracy of electronic adverse drug event detection. JAMIA 2012;29:31-38.

3. Lucado J, et al. Medication-related adverse outcomes in U.S. hospitals and emergency departments, 2008. HCUP Statistical Brief \# 109. AHRQ, 2011.

4. Handler SM, et al. A systematic review of the performance characteristics of clinical event monitor signals used to detect adverse drug events in the hospital setting. JAMIA 2007;14:451-8.

\section{*Ralph J. Coates}

E-mail: rjc5@cdc.gov 